\title{
Paradigmas para la enseñanza de las ciencias sociales en las escuelas de medicina*
}

\author{
Paradigms for the teaching of social sciences in the medical \\ schools
}

\author{
Juan César García †**
}

\section{RESUMEN}

En el presente trabajo se exponen algunos de los problemas de la enseñanza de las ciencias sociales en las escuelas de medicina, y se propone la aplicación de modelos pedagógicos como una forma de mejor enseñanza. La planificación de la enseñanza de cualquier disciplina en una carrera universitaria no puede hacerse sin tomar en cuenta los modelos que guían la enseñanza general y se incorporan las disciplinas específicas. Hasta ahora los planes de estudio no indican el marco conceptual unificador. Los planes de estudio de la mayoría de las escuelas de las profesiones de la salud en la América Latina separan las ciencias básicas de las clínicas, así como los diferentes niveles de prevención. Considerando las ventajas de una enseñanza integrada en los aspectos mencionados, se propone el uso de modelos o paradigmas educacionales integrados. Se presentan los paradigmas de Leavell y Clark como ejemplo de modelos educacionales que podrán emplearse al diseñar planes de estudio para las profesiones de la salud, y se propone uno para la enseñanza de las ciencias sociales.

Palabras clave: Ciencias sociales, escuelas de medicina, modelos integrados.

\begin{abstract}
Some of the problems in teaching of social sciences at the medical schools were presented, together with the proposal of educational models to improve teaching in this field. The teaching of a discipline in a university career can not be planned without taking the guiding models of the general education into account, and then the specific disciplines are added. Up to the present, curricula do not show the
\end{abstract}


unifying conceptual framework needed for this. Most of the health profession schools' curricula in Latin America separate basic sciences from clinical sciences as well as the different prevention levels. Taking into consideration the advantages of integrated teaching in the above-mentioned aspects, the use of integrated educational models or paradigms was put forward. Leavell and Clark's paradigms were provided as examples of educational models that might be used to design health profession curricula; at the same time, another model was suggested for the social sciences.

Key words: Social sciences, medical schools, integrated models.

\section{INTRODUCCIÓN}

La enseñanza de cualquier disciplina específica en una carrera universitaria plantea problemas que rebasan sus límites. Uno de estos problemas se refiere a la unidad programática que debiera tener la enseñanza de una profesión. La conformidad con este principio supone un enfoque totalmente distinto de la estrategia de la enseñanza para formar un especialista o un profesional. Lamentablemente esta distinción no siempre se observa en la realidad y así, es común encontrar que para formar médicos se enseña, por ejemplo, bioquímica o sociología, en igual forma que para preparar especialistas en la materia.

El desempeño de las profesiones de la salud (medicina, odontología, enfermería y otras afines) se basa fundamentalmente en la aplicación del conocimiento científico a la solución de problemas de salud en el hombre, y por tal razón, se considera a los integrantes del equipo médico como expertos en un área limitada del conocimiento especializado -que requiere, al igual que otras profesiones, un período largo de adiestramiento formal- y no como individuos poseedores de un conocimiento general. Parecería obvio, en consecuencia, que las instituciones donde se forman estos profesionales dieran máxima importancia a la enseñanza de las disciplinas directamente relacionadas con el desempeño de la ocupación y al adiestramiento en la aplicación del conocimiento. Pero éste no es el caso. El hecho de que en las últimas décadas los profesionales de la salud no hayan tenido en cuenta, en el ejercicio de su trabajo, al hombre como un todo, ha inducido a incorporar las disciplinas humanísticas en los planes de estudio. Esta tendencia parte del supuesto de que todo "hombre culto o poseedor de conocimientos generales" adopta una "actitud tolerante y humana"; esta relación, sin embargo, no parece tener una base empírica que la sustente.

En realidad, el defecto de la formación profesional estriba más bien en no proporcionar a los futuros profesionales conocimientos psico-socio-culturales que les permitan llenar su cometido en una forma más eficiente y comprensiva. Aplicar abstracciones y leyes generales en la solución de fenómenos concretos y particulares, es el problema más importante de la educación médica. El esquema clásico de enseñanza se ha limitado a impartir a los alumnos, durante los primeros años, conocimientos sobre ciencias básicas (fenómenos de carácter general) y posteriormente, enfrentarlos con la realidad del hombre enfermo (fenómenos de carácter particular). En ambos ciclos poco o nada se hace para que el alumno adquiera la habilidad de relacionar ambos niveles $y$, consecuentemente, los 
profesores de las ciencias básicas se quejan del empirismo de los clínicos, y los clínicos, a su vez, del conocimiento poco práctico que recibe el estudiante durante sus estudios en los años básicos.

Un ejemplo de lo que puede suceder cuando las abstracciones se enseñan sin la aplicación que debe dárseles en una situación específica, es el siguiente: en clínica médica el alumno tiene la oportunidad de comprobar que la disnea en un paciente con insuficiencia cardiaca congestiva aumenta con la posición decúbito. La observación clínica da oportunidad para relacionar estos hechos con los mecanismos generales que la explican y que son aprendidos en el ciclo básico; sin embargo, esta oportunidad no siempre se utiliza. Aun más, si ello se intentase, estaría restringida porque, en mayor grado, tales conocimientos han sido olvidados y recordarlos requiere un esfuerzo adicional.

Visto el proceso a la inversa, se comprueba que en las ciencias básicas el aprendizaje de fenómenos de carácter general se dificulta y se olvida con más facilidad cuando no se relacionaron fenómenos de carácter particular como los confrontados en la actividad clínica.

Pareciera aconsejable, por consiguiente, que los conocimientos, tanto de carácter general como particular, se enseñen conjuntamente, dedicando gran atención al desarrollo de destrezas y técnicas para definir situaciones nuevas y aplicar en forma adecuada el conocimiento general a lo particular. Tal como lo expresa Bogas ${ }^{1}$ con referencia a la educación general, "el estudiante descubre, a través de la solución de los problemas reales de la población, la importancia no solo de las destrezas y la información, sino también de las ideas y principios que deben guiarlo en el establecimiento y logro de metas."

Con el fin de obviar tal problema, han surgido dos tipos de solución: el de los reformistas y el de los revolucionarios.

Por mucho tiempo los reformistas han venido tratando de armonizar el ciclo de las ciencias básicas con el clínico mediante la integración vertical y horizontal, la cual, en la mayoría de los casos, se limita a transferir conocimientos de un ciclo a otro, sin desarrollar habilidad alguna para relacionar niveles de abstracción y de generalización. Los revolucionarios, por su parte, proponen eliminar el esquema clásico que separa el ciclo de las ciencias básicas del clínico, introduciendo simultáneamente el aprendizaje de lo abstracto y general y su aplicación a lo concreto y particular. El uso de paradigmas o modelos en la enseñanza y la aplicación de los principios de complejidad creciente y de refuerzo mediante la repetición, contribuirán a hacer factible esta proposición.

El término "Paradigma" viene del griego mostrar, manifestar, y lo definiremos como la presentación de un fenómeno o acontecimiento, atendiendo solo a sus líneas o caracteres más significativos. ${ }^{2}$

En un paradigma o modelo, la representación de un proceso se hace generalmente en forma gráfica, dibujando, por ejemplo, sus distintas fases como casilleros y la relación entre ellas como líneas. La representación gráfica permite formarse fácilmente una idea de conjunto de un fenómeno dado. Otra de las ventajas de un paradigma es que tiene un cierto nivel de generalidad que permite contar con un marco general de referencia aplicable a situaciones distintas dentro de un área semejante. ${ }^{2-4}$

En resumen, los paradigmas o modelos se usan como equivalentes esquemáticos de un fenómeno generalmente amplio y complejo. Los paradigmas suelen usarse en pedagogía porque se apoyan en tres principios educacionales: 1) "el hombre 
aprende mejor lo desconocido cunado parte de lo conocido", y los paradigmas subrayan lo conocido y sus implicaciones; 2) "el conocimiento se aprende mejor cuando se enseña en forma organizada y relacionada", y los paradigmas son generalizaciones que relacionan y organizan (sistematizan) hechos específicos y aislados; 3 ) "las abstracciones se aprenden más fácilmente cuando están relacionadas con fenómenos concretos", y los paradigmas son entidades concretas de fenómenos abstractos que, además, vinculan hechos específicos con abstracciones. En investigación, los modelos o paradigmas se usan con relativa frecuencia con el fin de proponer nuevas hipótesis o señalar vacíos en la investigación de un fenómeno que de otra forma no serían perceptibles.

Las ventajas evidentes que tienen los paradigmas no deben hacer perder de vista, sin embargo, los peligros potenciales de su uso. Así, el paradigma, que es una representación esquemática e hipotética de la realidad, está constituido por elementos o rasgos ajenos al fenómeno que se desea representar o simbolizar. Estos errores, que se pueden cometer al usar modelos, pueden impedir la adquisición de nuevos conocimientos o facilitar el conocimiento errado.

Dos principios se hayan ligados al uso de los modelos o paradigmas: el de complejidad creciente y el de refuerzo por repetición. Un mismo modelo o paradigma se podría aplicar repetidamente para enseñar diferentes fenómenos particulares y concretos, por ejemplo, diversas entidades patológicas. El modelo o esquema sería siempre el mismo y se repetiría tantas veces como entidades particulares se estudiarán, permitiendo al alumno el descubrimiento de "lo común" en una serie de fenómenos, y proporcionándole una herramienta conceptual que le permitiría solucionar con mayor facilidad un problema desconocido. Esta repetición difiere de la que habitualmente se encuentra en el sistema tradicional, en la cual sólo se reiteran hechos dispersos y no existe un marco conceptual lo suficientemente general para explicar fenómenos distintos.

Un paradigma educacional rinde sus mejores resultados cuando se aplica a fenómenos que paulatinamente se vuelven cada vez más complejos. Se entiende por fenómeno de alta complejidad aquel que requiere un gran número de factores o variables para su explicación.

Los problemas que confronta la enseñanza de asignaturas particulares son los mismos que afectan la educación médica en general, y es por eso que se estima pertinente la aplicación de algunas de las innovaciones comentadas, especialmente en lo que se refiere a la trasmisión de conocimientos de las ciencias sociales. Se espera con ello aumentar la eficiencia y la amplitud de la acción médica, ya que es imposible y poco recomendable enseñar al estudiante de las profesiones de la salud todos los conocimientos acumulados por aquellas. El criterio aconsejable en la selección de tal trasmisión es la aplicación actual y futura de dichos conocimiento en la solución de los problemas que el médico confronta en su ejercicio diario. Los modelos que permiten un mejor y más rápido aprendizaje y el empleo del principio de complejidad creciente, podrán contribuir a seleccionar los conocimientos que se quieren trasmitir, así como ordenarlos de modo que se facilite su aprendizaje y se garantice su aplicación práctica.

\section{LA ENSEÑANZA DE LAS CIENCIAS DE LA CONDUCTA EN FACULTADES DE LAS CIENCIAS DE LA SALUD}

Desde el momento en que establecimos que las profesiones de la salud aplican conocimientos científicos, nos hemos comprometido con un enfoque particular de la enseñanza del conocimiento de las ciencias naturales y sociales pertinentes a 
dichas profesiones. En consecuencia, habría que considerar tres elementos fundamentales en el planeamiento de su enseñanza:

1. El criterio orientador en la selección de conocimientos apropiados; este puede enunciarse así: Debe enseñarse todo marco conceptual que contribuya a un mejor desempeño, actual y futuro, de las actividades de las profesiones médicas.

Este criterio es difícil de emplear en la enseñanza de las ciencias sociales, en parte, porque estas todavía no cuentan con una experiencia amplia de aplicación y, en buena medida, por la dificultad de manejar las variables sociales para producir cambios deseables. Si bien existe un vasto conocimiento sobre los factores que determinan ciertas áreas del comportamiento humano, estos no son de fácil manipulación, y si lo son, muchas veces, por razones éticas, no pueden ser llevados a la práctica. Por otra parte, las ciencias sociales no han desarrollado lo suficiente las teorías de cambio conductual y social; y de ser el cambio el objetivo fundamental de todo modelo de aplicación de conocimiento científico, debe ser también el eje de la actividad de las profesiones médicas.

Lo expresado no debe llevarnos a pensar que no se ha hecho nada por aplicar los conocimientos de las ciencias sociales a la solución de problemas. Por ejemplo, en el campo de la educación, el aporte de las ciencias sociales ha sido fundamental y útil. En medicina las posibilidades son grandes, como lo demuestran los ensayos de aplicación llevados a cabo por profesionales médicos y por científicos sociales.

2. Otro elemento que debe tenerse en cuenta en el planeamiento de la enseñanza, es el enfoque que se utilizará en la definición y solución de problemas médicos, donde las variables psico-socio-culturales son de importancia. Gouldner, ${ }^{5}$ describe dos tipos: el de ingeniería y el clínico.

En el enfoque de ingeniería el problema es definido por el cliente y aceptado por el científico social. En el enfoque clínico, por el contrario, el científico social hace el diagnóstico independientemente de la forma en que el cliente presenta el problema, pues asume que el cliente tiene dificultad en plantear sus propios problemas, incapacidad que puede estar motivada por el poco deseo de querer explorarlos a fondo o remediarlos. Por consiguiente, en el enfoque clínico la forma de trabajo del científico social se asemeja a la del médico. Como ejemplo, Gouldner transcribe la descripción que Emile Durkheim hace de la actividad médica: "un hombre enfermo interpreta erróneamente los síntomas que siente, los cuales atribuye, frecuentemente, a causas que no son las verdaderas. Pero estos síntomas, tal como son expresados, son de interés, y el clínico los anota con gran cuidado y seriedad. Ellos son elementos en el diagnóstico, y muy importantes... el médico presta atención al lugar donde el paciente siente los síntomas y cuándo empezó a asentirlos". Las profesiones médicas, por su prestigio y amplia experiencia en la solución de problemas prácticos, tienden a imponer a los científicos sociales su definición de los problemas. En otras palabras, asumen que los sociólogos, psicólogos y antropólogos deben trabajar sobre las bases de un enfoque de ingeniería. Esta situación se agrava en las instituciones docentes donde, por lo general, el científico social se deja influir por los médicos y llega a convertirse en un técnico en diseño de cuestionarios y en estadística social.

La aportación del científico social será fructífera sólo si en su conducta profesional utiliza el enfoque clínico, es decir, si en la solución del problema toma en cuenta la posible resistencia del cliente.

3. El tercer elemento importante en el planeamiento de la enseñanza está relacionado con su metodología. Como las dificultades que confronta la enseñanza 
médica, especialmente las referentes a la aplicación de conocimientos, suelen ser semejantes a las de las ciencias sociales, es de suponer que en su enseñanza se sigan las mismas recomendaciones que para el plan de estudios en general. Así, se deberá adoptar el enfoque revolucionario, caracterizado por la enseñanza simultánea de los principios y las abstracciones generales y de su aplicación a los problemas médicos. Si se adoptara el enfoque tradicional, es decir, la enseñanza de conocimientos y conceptos generales de las ciencias sociales en los primeros años de estudio y aplicación en los años clínicos, no se lograría el aprendizaje de la aplicación y no se motivaría suficientemente al alumno.

El estudiante de medicina centra su interés en sus relaciones con el paciente, y todo lo que se aparte de esto no le motivará suficientemente.

Una enseñanza que gire alrededor de la aplicación práctica, dentro del área de interés del alumno, tendrá mayores posibilidades de aceptación y comprensión de un marco conceptual general y abstracto.

\section{PARADIGMAS PARA LA ENSEÑANZA DE LAS CIENCIAS SOCIALES}

Después de señalar la necesidad de enseñar las ciencias sociales estrechamente ligadas a los problemas médicos, y específicamente a las actividades de las profesiones de la salud, queda por seleccionar paradigmas que, además de auxiliar en la enseñanza, cumplan con estos objetivos. En la selección de paradigmas se tuvieron en cuenta los siguientes criterios: a) que los modelos hubiesen sido elaborados por la profesión médica y, por lo tanto, que fueran conocidos por esta; b) que describieran el proceso enfermedad-salud y las acciones médicas en forma general e integral, de manera que permitieran la incorporación del mayor número de variables psico-socio-culturales pertinentes.

Se seleccionaron como paradigmas básicos los elaborados por Leavell y Clark, ${ }^{6}$ a los cuales se agregaron otros más específicos y originados en las ciencias sociales. Esta selección no significa que no haya otros paradigmas de igual o mejor eficacia educacional.

\section{Paradigma de la historia natural de la enfermedad}

Leavell y Clark propusieron sus paradigmas con la intención de desarrollar un punto de vista y un método de enfocar la promoción de la salud y la prevención de la enfermedad en su sentido más amplio.

El primero titulado, "La historia natural de la enfermedad", se refiere al proceso de enfermedad. El esquema puede ser aplicado a todo tipo de enfermedad y, por consiguiente, sirve para ordenar el conocimiento existente, indicar los vacíos en el mismo y promover nuevos hallazgos mediante la investigación; es decir, que el paradigma no es una teoría sobre la enfermedad, sino que constituye una forma de pensar que lleva a la construcción de un sistema teórico (figura 1). 


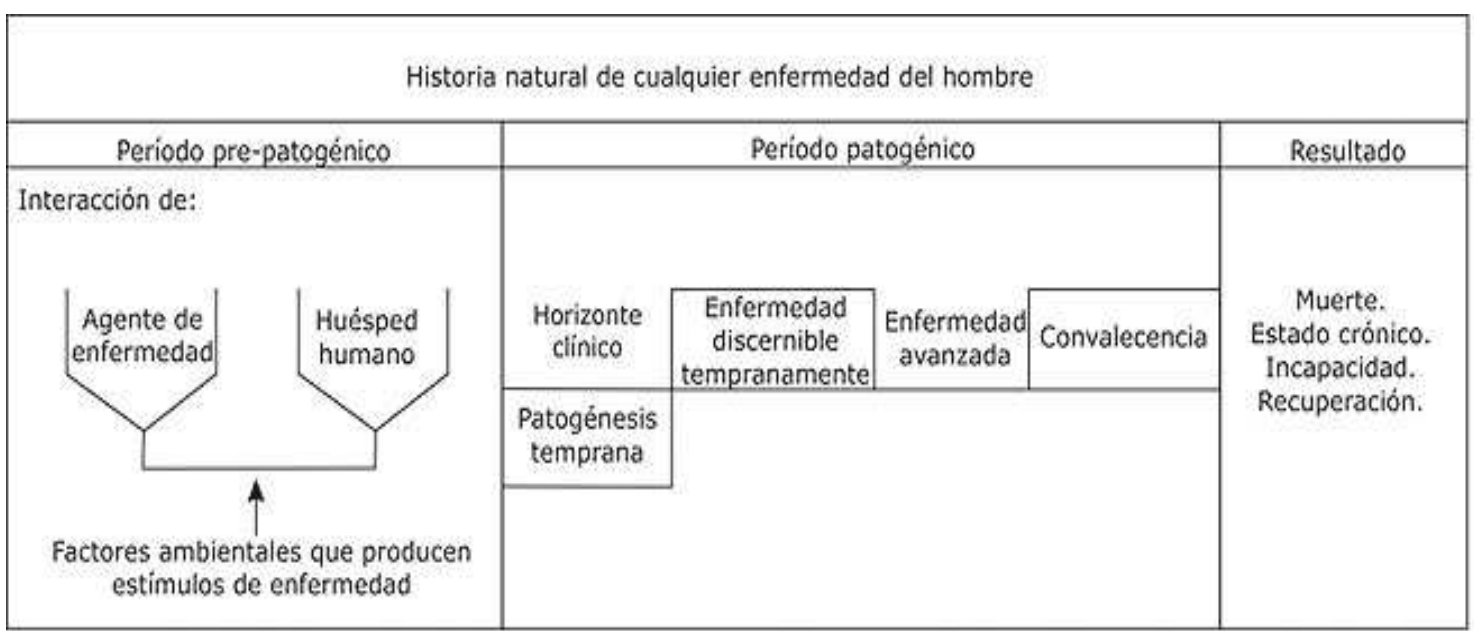

Fuente: Leavell y Clark. Preventive Medicine for the Doctor in his Community. McGraw-Hill book Company, New York, 1965, pag. 18.

Fig. 1. Paradigma No. 1. La historia natural de la enfermedad.

La enfermedad concebida como un proceso, presupone una serie de etapas sucesivas que Leavell y Clark agrupan en dos grandes períodos: el pre-patogénico y el patogénico. En el período pre-patogénico se establece la interacción entre el agente potencial de la enfermedad, el huésped y los factores ambientales. Por ejemplo, en el caso de la malaria, el reservorio Plasmodium es el hombre, pero nuevas infecciones dependen de las condiciones del agente determinante de la enfermedad y del ambiente que rodea al hombre (la presencia del mosquito Anopheles, condiciones ambientales satisfactorias para su propagación y los hábitos y costumbres del hombre con referencia a mecanismos de protección).

El período patogénico se inicia con el estímulo que originan los cambios en la estructura y el funcionamiento del organismo humano, y termina con su recuperación, incapacidad o su muerte. Este período comprende varias etapas: a) patogénesis temprana; b) enfermedad discernible tempranamente; c) enfermedad avanzada; d) convalecencia y e) resultado final (recuperación, estado crónico, incapacidad o muerte). La etapa de patogénesis temprana se caracteriza por las reacciones y cambios que se producen en los tejidos y en el funcionamiento del organismo a consecuencia del estímulo que ocasiona la enfermedad. La presencia de signos y síntomas que permiten el diagnóstico clínico marca el pasaje a las etapas siguientes.

Las disciplinas biomédicas y las ciencias sociales contribuyen al conocimiento de la enfermedad. Las últimas, aplicadas al estudio de la enfermedad, tienden a esclarecer los factores psico-socio-culturales que influyen tanto en la producción como en la propagación de la enfermedad en la población. Estas disciplinas, si bien de reciente origen, constituyen ya un recurso indispensable en la solución de problemas en la práctica diaria del médico.

El paradigma de "historia natural de la enfermedad" es útil y sencillo; sin embargo, contiene supuestos que pueden ser discutibles. Explicaremos estos supuestos y los aceptaremos provisoriamente sin una crítica profunda, aunque estamos conscientes de sus peligros.

El primer supuesto del esquema es la consideración de la enfermedad como un proceso y no como un estado; en otras palabras, es un fenómeno dinámico. En este sentido, enfermedad es un conjunto de fases sucesivas; por ejemplo, la tuberculosis en su desarrollo presenta diversas etapas o fases: 
a) La fase infecciosa, que sigue directamente a la implantación de los bacilos de la tuberculosis en los tejidos, se conoce como tuberculosis primaria o primoinfección tuberculosa. En la mayoría de los pacientes, la tuberculosis primaria es curable y su progreso puede detenerse.

b) En algunos pacientes, probablemente menos del $10 \%$, el complejo inicial progresa y se disemina, por lo que puede producir muchas formas de tuberculosis.

c) La tuberculosis puede tener también una fase de reinfección que sigue a la reactivación de la tuberculosis primaria o a la reinfección de un sujeto previamente expuesto.

Las manifestaciones clínicas de la tuberculosis en cada fase son muy variadas en cuanto al carácter, la localización y la extensión de las lesiones, hecho que ha llevado a ciertos autores a dudar de la validez de agrupar las diversas fases bajo una misma denominación. En el esquema, en cambio, se supone que existen conexiones entre fase y fase, lo cual podría justificar su clasificación bajo un mismo proceso.

En el segundo supuesto del esquema, sin embargo, se sostiene que las conexiones aludidas no son de tipo casual. Por ejemplo, la fase de la tuberculosis primaria no es causa de la fase de la tuberculosis secundaria. Cada fase es el resultado de procesos internos y de factores externos, y la conexión entre las fases previas y las posteriores es de tipo genético y no causal.

La denominación de "historia natural de la enfermedad" dada por Leavell y Clark al esquema, refleja el tercer supuesto, el cual postula que la enfermedad sigue una trayectoria más o menos definida, es decir, que ya en las primeras fases existen los elementos que aparecen en fases posteriores. Este fluir irremediable solo podrá ser interrumpido por la acción preventiva.

\section{Paradigma de las acciones preventivas}

Si el propósito de la medicina es interrumpir el curso de la enfermedad, es necesario entonces conocer todos los factores que causan o mantienen el trastorno. Con un mejor conocimiento de las variables que intervienen en la historia natural de la enfermedad, se podrán aplicar medidas efectivas, a fin de interrumpir el curso en cualquiera de sus fases. Sin embargo, en el caso de muchas enfermedades todavía no se ha llegado al pleno conocimiento de tales variables. Así, los factores sociales, por ejemplo, que intervienen en los períodos pre-patogénicos y patogénicos, no han sido estudiados a fondo y, por lo tanto, no se sabe aún como manejarlos para prevenir los trastornos patológicos. El éxito de la acción médica dependerá del conocimiento que se tenga de los factores que intervienen en la producción y el curso de la enfermedad.

Teóricamente, esta se puede prevenir en cualquiera de sus fases, dependiendo, como ya se dijera, de lo que se conozca sobre su historia. Así, en el período prepatogénico se puede prevenir mediante la promoción general de la salud y la protección específica contra el agente causal. Esto es lo que Leavell y Clark han llamado prevención primaria.

La prevención secundaria se refiere a la acción desplegada durante las primeras fases del período patogénico, y se puede lograr mediante un diagnóstico temprano y un tratamiento adecuado. 
La prevención terciaria incluye los mecanismos destinados a reducir la incapacidad y corregir los defectos (figura 2).

\begin{tabular}{|c|c|c|c|c|}
\hline \multicolumn{2}{|c|}{ Periodo pre-patogénico } & \multicolumn{3}{|c|}{ Periodo patógenico } \\
\hline \multicolumn{2}{|c|}{ Prevesción primara } & \multicolumn{2}{|c|}{ Prevención secundaria } & $\begin{array}{l}\text { Preventión } \\
\text { terelaria }\end{array}$ \\
\hline $\begin{array}{l}\text { Promoclin de } \\
\text { la salud }\end{array}$ & $\begin{array}{l}\text { Protección } \\
\text { especifica }\end{array}$ & $\begin{array}{l}\text { Diagnóstice y } \\
\text { tratamiento } \\
\text { temprano }\end{array}$ & $\begin{array}{l}\text { Limitaciden de la } \\
\text { incapacidad }\end{array}$ & Rehabilitación \\
\hline $\begin{array}{l}\text { Educación sanitaria. } \\
\text { Buenos estándares de } \\
\text { nutrición ajustados a } \\
\text { las diferentes fases } \\
\text { de desarrollo. } \\
\text { Atención al desarrollo } \\
\text { de ia personalidad. } \\
\text { Provistón de condioiones } \\
\text { adecuadas decasa, } \\
\text { recreoción ycondioiones } \\
\text { de trabajo. } \\
\text { Genética. } \\
\text { Exámenes periódicos } \\
\text { selectivos. }\end{array}$ & $\begin{array}{l}\text { Uso de inmunizaciones } \\
\text { especificas. } \\
\text { Atención a la higiene } \\
\text { personal. } \\
\text { Uso de saneamiento } \\
\text { ambiental. } \\
\text { Protección contra los } \\
\text { resgos coupacionales. } \\
\text { Protecoión contra } \\
\text { accidentes. } \\
\text { Uso de nutrientes } \\
\text { específicos. } \\
\text { Protección contra } \\
\text { carcinógenes. }\end{array}$ & $\begin{array}{l}\text { Medidas para } \\
\text { encontrar casos } \\
\text { incividuales y de masa } \\
\text { Exdimenes seiectivos. } \\
\text { Encuestas de selección } \\
\text { de casos. } \\
\text { objetivos: } \\
\text { Curar y prevenir ta } \\
\text { enfermedad. } \\
\text { Prevenir la difusión de } \\
\text { enfermedades } \\
\text { comunicables. } \\
\text { Prevenir } \\
\text { complicaciones y } \\
\text { secuelas. } \\
\text { Accrtar el periodo de } \\
\text { incapscidad. }\end{array}$ & $\begin{array}{l}\text { Adecuado tratamiento } \\
\text { para detener is } \\
\text { enfermedad y prevenic } \\
\text { futuras complicaciones } \\
\text { ysecuelas. } \\
\text { provision defacilidades } \\
\text { conei finde limitar } \\
\text { incapacidady } \\
\text { prevenir la muerte }\end{array}$ & $\begin{array}{l}\text { Provisión de facilidades } \\
\text { hospitalarias y } \\
\text { comunitarias para } \\
\text { adiestramiento y } \\
\text { educación, con el fin de } \\
\text { usar al máximo las } \\
\text { capacidades remanentes. } \\
\text { Educación del público y } \\
\text { de la industria para } \\
\text { utilizar al rehabilitado. } \\
\text { Trabajo como terapiaen } \\
\text { los hospitales. } \\
\text { Ubicación selectiva. }\end{array}$ \\
\hline
\end{tabular}

Fuente: Leavell y Clark, Preventwe Medicine for the Doctor in his Community. McGraw-Hill book Company, New York, 1965, pag. 18.

Fig. 2. Paradigma No. 2. Niveles de aplicación de medidas preventivas en la historia naturai de la enfermedad.

En resumen, se pueden distinguir cuatro niveles de aplicación de medidas preventivas en la historia natural de la enfermedad: 1) Promoción de la salud; 2) Protección específica; 3) Diagnóstico y tratamiento temprano; y 4) Rehabilitación.

\section{Paradigmas de las conductas preventivas del equipo médico, de la población y del contexto socio-cultural}

Una vez conocida la historia natural de la enfermedad y las medidas preventivas adecuadas, procede conocer y saber utilizar los factores que influyen decisivamente en la efectividad de estas medidas.

Los factores que afectan el nivel de aplicación de las medidas son de orden económico, social y de organización. Aquí nos interesan particularmente aquellos elementos estudiados por las ciencias sociales que, en una forma u otra, facilitan o impiden las acciones preventivas.

En la prevención primaria de la poliomielitis, por ejemplo, la protección específica adecuada es la inmunización, la cual debe llevarse a cabo con la participación activa y conjunta de los organismos médicos y de la población. Sin embargo, muchas veces el nivel requerido de vacunación no se alcanza por diversas razones, entre otras, la resistencia o falta de interés por parte de la población, la organización deficiente de las instituciones médicas, o la carencia de servicios médicos. Estos y muchos otros factores clasificados como psico-socio-culturales, impiden llevar a cabo una acción efectiva.

Los factores estudiados por las ciencias sociales que tiene relación directa con la toma de decisión y la ejecución de medidas preventivas, se pueden agrupar en tres amplias categorías: los que tienen que ver con la profesión médica, los relacionados con la población a la que va destinada la acción preventiva, y los que influyen en el marco económico y socio-cultural que engloba las dos anteriores. Por ejemplo, en la prevención del cáncer podemos reconocer factores psico-sociológicos que impiden que los médicos hagan un diagnóstico temprano de la enfermedad a pesar 
de estar en condiciones de realizarlo, o que la población en peligro no solicite a tiempo atención médica, o que los servicios médicos no estén a disposición de toda la población.

Los factores relacionados con la población y que tienen que ver directamente con la aplicación eficaz de medidas preventivas, han sido estudiados por diversos autores $^{7,8}$ bajo la denominación de "conducta preventiva del hombre en estado de salud y de enfermedad" (figura 3 ).

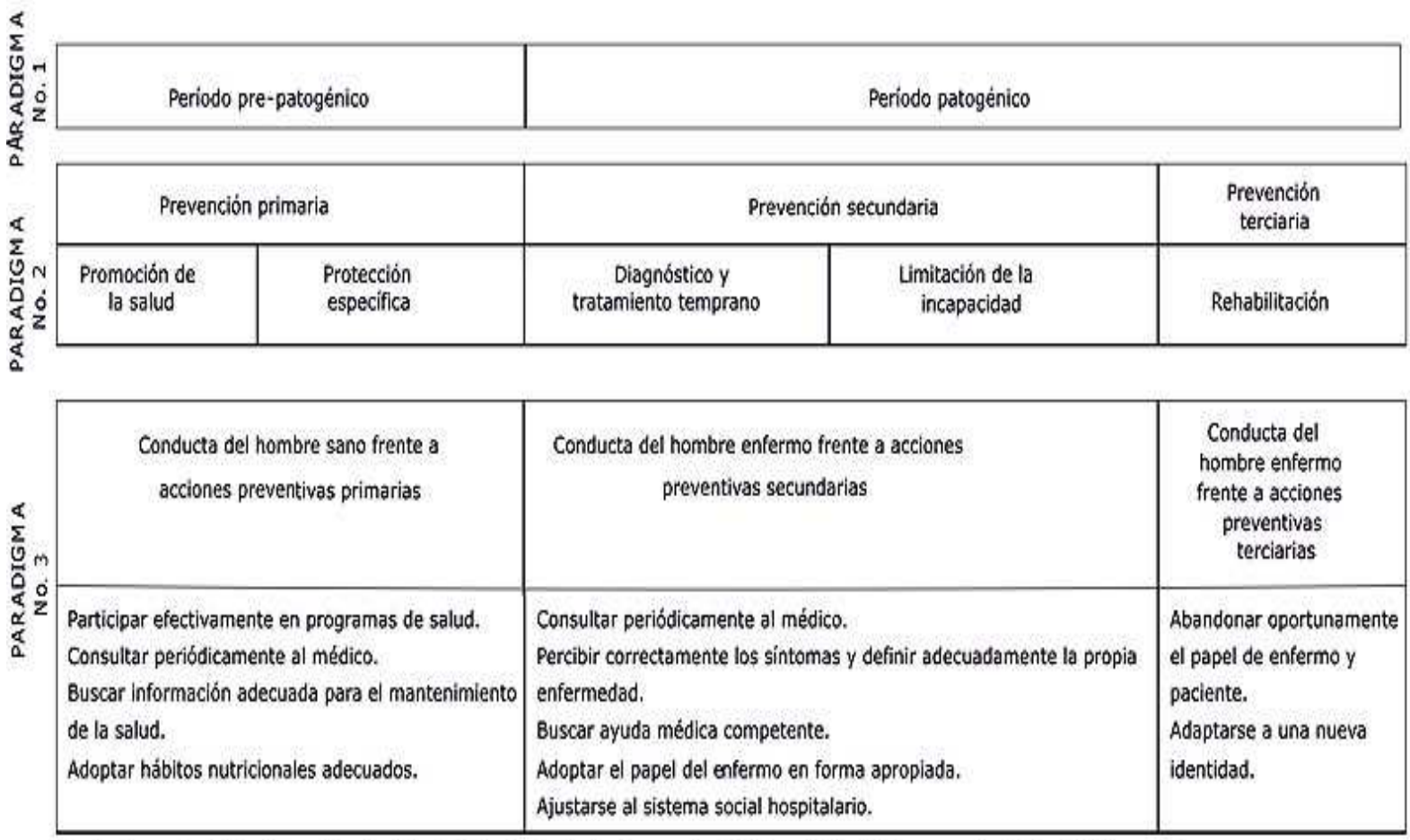

Fuente: Leavell y Clark. Preventive Medicine for the Doctor in his Community. McGraw-Hill book Company, New York, 1965, pag. 18.

Fig. 3. Paradigma No. 3. Conducta preventiva en estado de salud y de enfermedad.

La clasificación de la conducta preventiva en estado de salud y de enfermedad repite, como puede verse en la figura 3 , las categorías utilizadas por Leavell y Clark para los niveles de aplicación de las medidas preventivas. Esta correspondencia tiene la ventaja de destacar la relación entre la fase de la enfermedad, la acción preventiva apropiada del caso y el papel de la conducta humana en la realización de dicha acción.

Cada una de las categorías de la conducta preventiva presenta un conjunto de decisiones críticas que el individuo debe confrontar. Desde el punto de vista médico, una o varias decisiones incorrectas pueden favorecer el desarrollo de la enfermedad hacia un estado de mayor gravedad. Todas estas decisiones están relacionadas con los diferentes niveles de aplicación de las medidas preventivas. En la figura 3 , se mencionan, bajo las categorías respectivas, algunas de las conductas específicas de carácter positivo para detener el curso de la enfermedad. Cuando las conductas son opuestas a las señaladas, habrá mayores posibilidades de que la enfermedad progrese hasta llevar al individuo a la incapacidad permanente o a la muerte.¿Qué importancia tiene la conducta preventiva de la población? La conducta de la población, al igual que la del equipo médico, tiene un impacto directo sobre el grado de cobertura de la atención médica. Se entiende por cobertura, la proporción de individuos de una población dada que está bajo cuidado médico, y el ideal es difícil de alcanzar y dependerá, en cierta medida, de la conducta de la población. Estudios sobre poblaciones aparentemente sanas han demostrado que cerca del 
$75 \%$ tienen algún trastorno físico o clínico y que solo el $25 \%$ de sus enfermos se hallan bajo cuidado médico. ${ }^{9}$ Si se considera que la circunstancia de estar bajo cuidado médico depende en parte del individuo mismo, es obvia la importancia de su conducta cuando se desea aumentar la demanda de atención médica. La conducta no solo es importante para consultar al médico, sino también para mantenerse bajo su cuidado.

En términos del paradigma presentado, podemos decir que la conducta del individuo con respecto a la cobertura es más decisiva cuando aquél se encuentra sano, en estado de transmisión entre salud y enfermedad y en el momento en que se deja de ser paciente.

La conducta de los integrantes del equipo médico, por su parte, aumenta en importancia con respecto a la cobertura a medida que se establece la relación equipo médico-paciente. Esto nos lleva a examinar el siguiente paradigma: "conducta de los integrantes del equipo médico frente a la enfermedad y a la salud."

Desde la antigüedad se viene señalando la influencia que tiene la conducta médica sobre las acciones de los pacientes. El Ilamado "arte" de la medicina era más que todo el manejo intuitivo de variables psico-socio-culturales que tenían repercusión sobre el comportamiento del individuo. Según los antiguos maestros, este arte no se enseñaba sino que era más bien una condición innata del individuo, o se adquiría ya fuera imitando ciertos modelos de buen clínico o por experiencia. Hoy día, con un mejor conocimiento de estas variables y de su importancia, tanto su estudio como el adiestramiento en su manejo, deberían introducirse en la enseñanza médica. En este modelo, la conducta también se clasifica en tres categorías: la relacionada con las acciones preventivas primarias, la que tiene que ver con las secundarias, y la relativa a las acciones preventivas terciarias. El paradigma que se presenta en la figura 4 podría servir de guía en la enseñanza de estas categorías. 
Revista Cubana de Salud Pública; 2010 36(4)371-380

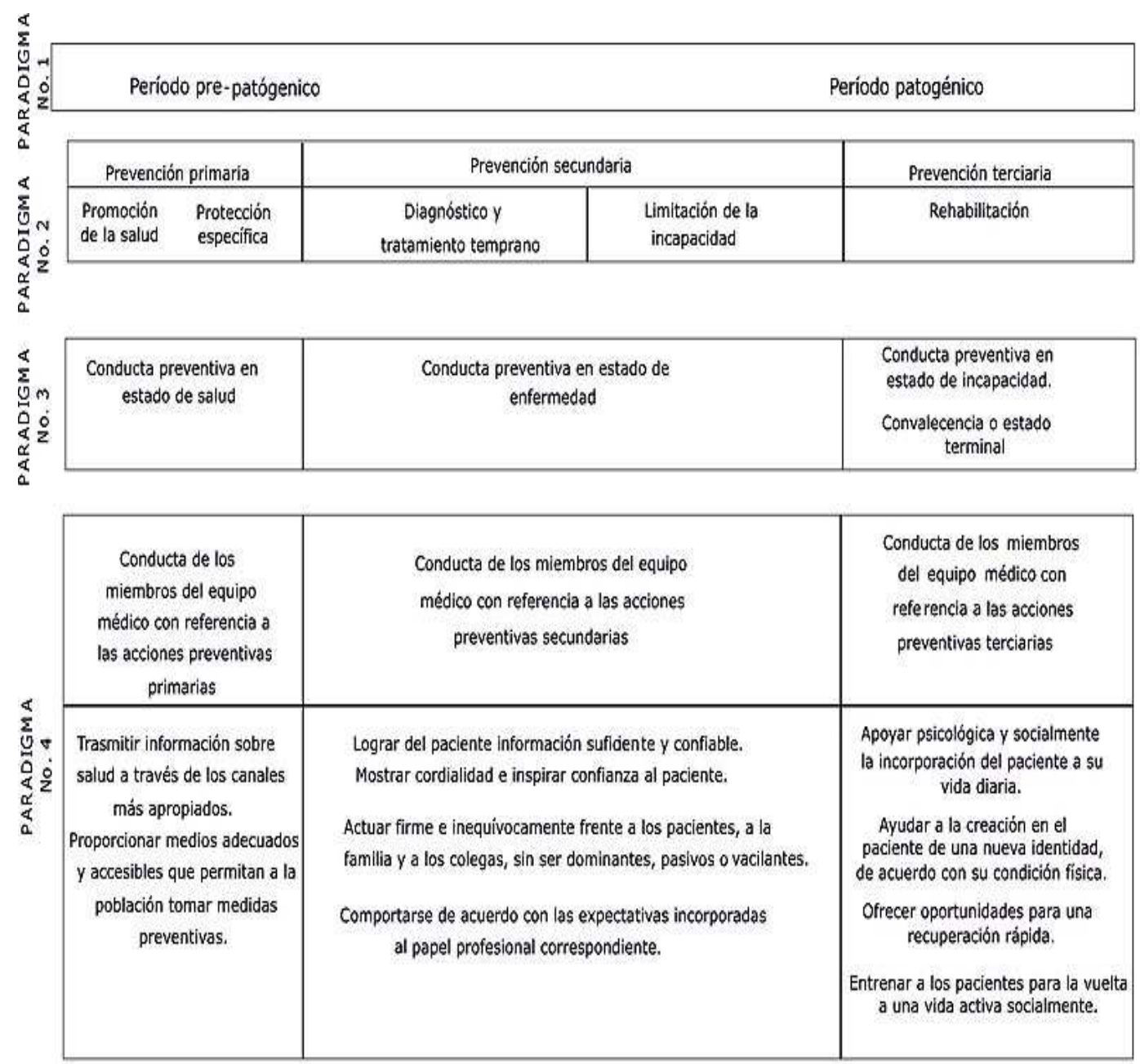

Fuente: Leavell y Clark. Preventive Medicine for the Doctor in his Community. McGraw-Hill book Company, New York, 1965, pag. 18.

Fig. 4. Paradigma No. 4. Conducta del equipo médico frente al hombre enfermo y sano.

Sin embargo, la conducta de la persona enferma o la sana en peligro de enfermedad y la de los profesionales, no ocurre en un vacío; ambas se mueven en un contexto que las determina en gran medida. El contexto político, el grado de dependencia económica de los países y el desarrollo histórico de las instituciones, no solo ayudan a explicar los problemas de salud de un país o región, sino también a entender la conducta de las poblaciones y del equipo médico frente a la salud y a la enfermedad.

En la figura 5 se integran los diferentes modelos analizados. Este conjunto de paradigmas deberá ser empleado por el estudiante en el análisis y solución de todo problema de salud. 


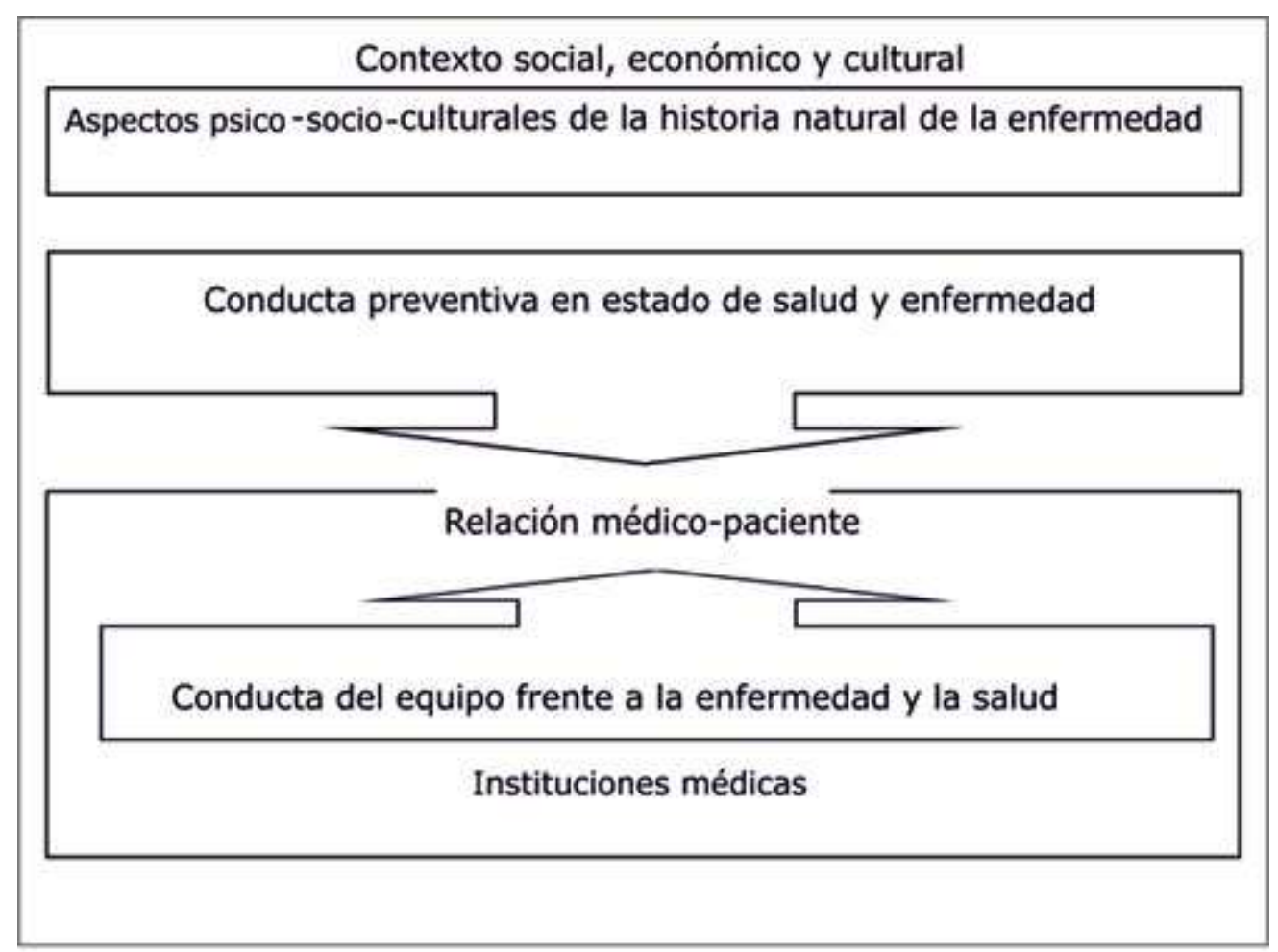

Fig. 5. Paradigma completo para la enseñanza de las ciencias sociales.

\section{APLICACIÓN DE LOS PARADIGMAS A LA ENSEÑANZA DE LA CARRERA MÉDICA}

Una de las características del uso de paradigmas en educación es que deberían aplicarse a todas las unidades del plan de estudios, es decir, a aquellas que se han designado tradicionalmente como asignaturas o materias.

El fraccionamiento del plan de estudios en materias es el resultado de la evolución de las disciplinas y no de una decisión pedagógica consciente. La crítica a la enseñanza por disciplinas o asignaturas no se restringe solamente al área médica. Así, autores como Bogas señalan, con respecto a la educación en general, que "los planes de estudios no deberían consistir de asignaturas, la escuela debería estructurarse en grupos de jóvenes que trabajen en "talleres" como un equipo.

Estos equipos deberían ser estimulados para que: a) identifiquen las necesidades o problemas de la población; b) seleccionen un determinado problema como foco de actividad; c) diseñen un plan para su solución y d) lleven a cabo las actividades que forman parte del plan. El estudiante, con este tipo de plan de estudio adquiere, en forma natural y normal y como parte del proceso de solución de problemas, una serie de habilidades y conocimientos.

Generalmente, unidades basadas en asignaturas o disciplinas, separan o desintegran aquellos aspectos de la enseñanza que más se desean integrar. Por lo tanto, para poder aplicar en la enseñanza modelos como el de Leavell y Clark, el plan de estudios debe estar dividido de suerte que se puedan emplear en todas las unidades. Existen, en este sentido, diversas formas de dividir el plan de estudios de forma tal que en cada unidad los estudiantes solucionen un problema de salud, guiados por paradigmas educacionales; por ejemplo, formando unidades derivadas de la estructura orgánica (aparatos y sistemas) o por tipo de agente productor de enfermedad (físico, biológico y otros). 
Una división del plan de estudio que tenga en cuenta los elementos discutidos, supone que en la enseñanza de una unidad deben intervenir especialistas de diversas disciplinas. Así, el sociólogo, el antropólogo y el psicólogo social participarían en una enseñanza íntimamente integrada con la de otros especialistas, e impartirían el conocimiento específico necesario para la comprensión de la historia natural de la enfermedad y de las acciones preventivas en todos sus niveles.

Esta forma de enseñanza es meta lejana para la mayoría de las escuelas de medicina de la América Latina y su aplicación supone un cambio radical del plan de estudios y de su estructura académica. Dada la imposibilidad de un cambio radical del plan de estudios, podría optarse por una solución transicional en el área de las ciencias sociales, transformando su enseñanza mediante la aplicación de paradigmas tales como los presentados en este estudio.

\section{REFERENCIAS BIBLIOGRÁFICAS}

1. Bogas Grace L. Education-The Great Obsesión. Monthly Review. 1970;2218-39.

2. Gage NL. Paradigms for Research on Teaching. En: Gage L, editor. Handbook of Research on Teaching. Chicago: Rand MacNally and Company; 1963.

3. Hill Joseph E, Kerber A. Models, Methods and Analytical Procedures in Education Research. Detroit, Mich.: Wayne State University Press; 1967.

4. Edwards JM. Creativity (II Social Aspects). En: Sills DL, editor. V 3 de la International Encyclopaedia of the Social Sciences. New York: The Macmillan Company y The Free Press; 1968.

5. Gouldner AW. Explorations in Applied Social Sciences. En: Gouldner AW, Miller SM, editors. Applied Sociology-Opportunities and Problems. New York: The Free Press; 1965.

6. Leavell HR, Clark EG. Preventive Medicine for the Doctor in His Community. New York: McGraw-Hill Book Company; 1965.

7. Suchman EA. Stages of Illnessand Medical Care. J Health \& Hum Behav. $1965 ; 6: 114-28$.

8. Mechanic D. Medical Sociology. New York: The Free Press; 1968.

9. White Kerr L. Patterns of Medical Practice. En: Preventive Medicine Duncan WC, Macmahon B, editores). Boston, Mass.: Little, Brown and Company; 1967.

\footnotetext{
*Trabajo preparado para los Seminarios de Ciencias de la Conducta en Escuelas de Medicina y utilizado en el "Taller de Educación en Ciencias de la Salud", Ilevado a cabo en Washington, D.C., del 18 de enero al 5 de marzo de 1971.

Tomado de: Educación Médica y Salud. 1971;5(2):130-48.

**Funcionario del Departamento de Desarrollo de Recursos Humanos, Oficina
} 
Sanitaria Panamericana, Oficina Regional de la Organización Mundial de la Salud, Washington, D.C. 